\title{
A Taylor-based plasticity model for orthogonal machining of single-crystal FCC materials including frictional effects
}

\author{
Eralp Demir
}

Received: 2 July 2007 / Accepted: 16 January 2008 / Published online: 4 March 2008

(C) Springer-Verlag London Limited 2008

\begin{abstract}
The purpose of this study is to explain the experimentally observed variations in cutting parameters during the machining of single-crystal materials. Fundamental relationships between crystal plasticity and machining are developed. The workpiece anisotropy stem from crystallographic differences are explained with a rateinsensitive Taylor plasticity model. A brief discussion of the applicability of Schmid-based models to machining processes is also presented. The periodic variations with changing crystal orientations observed in experimental studies are explained with the results of the proposed model for machining. The friction between the rake face of the tool and the material is introduced to the existing model. The applicability of concepts like Texture Softening Factor and Effective Taylor Factor in previous works are discussed. The specific energy of cutting is related to Taylor factor for better understanding of crystallographic effects.
\end{abstract}

Keywords Taylor factor C Crystal plasticity

Orthogonal cutting $\cdot$ Single-crystal FCC

\section{Introduction}

It is important to know how good the quality of a product will be prior to machining. In other words, under which cutting conditions and along which direction in the

This work was partially completed with Prof. Burak Ozdoganlar, Carnegie Mellon University, Mechanical Eng. Dept.

E. Demir $(\bowtie)$

Max-Planck-Institut fur Eisenforschung,

Max-Planck-Str. 1,

40074 Dusseldorf, Germany

e-mail: demir@mpie.de workpiece, how the quality of the machined product will be. Some experimental measures like cutting force, specific energy, chip morphology and shear angle can be used as a reference to give a of wealth information about the machined product. With recent developments in cuttingtool technology, tools having sizes smaller than grain size have now become available, highlighting the importance of single-crystal machining. The demand for a model explaining crystal-to-crystal differences as well as differences of machining single crystals along different crystallographic directions has arisen. Numerous studies on both single and polycrystal metals have elucidated the quality of machining being effected by the crystal orientation of the workpiece at small scales. Understanding the difference in cutting different orientations in the crystals has been a fundamental issue even for orthogonal planing, which is the simplest deformation case. However, just a few modeling efforts exist in literature that relate crystal orientation to machining performance. There has not yet been an explanation of the very low shear angles observed when machining single crystals against the Merchant minimum energy principle.

Orthogonal planning is the simplest machining operation and it could be easily extended to the other conventional machining processes like milling and drilling. It is a plane strain deformation where the width of cut is significantly large when compared to the depth of cut. The deformation is of simple shear type in which the amount of strain increases with decreasing rake angle (Eq. 1).

$\gamma=45-\frac{\alpha}{2}$

Primary shearing during machining occurs in a single plane and shear angle is a free parameter according to shear-plane assumption [1]. Merchant, maximizing shear stress at the shear plane, obtained a relationship for the 
shear angle $(\phi)$ (Eq. 2) in which $\alpha$ is the rake angle and $\beta$ is the tangent of the friction coefficient between the rake face of the tool and the chip. It could be easily seen that friction has a lowering effect on shear angle. This relationship ignores the dependency of both friction angle and resultant force on shear angle and it always gives shear angles close to $45^{\circ}$, which does not explain the very low shear angles observed. On the other hand, the shear plane solutions assume flow stress being constant which rules out the effects of strain-rate depend on cutting speed [2]. However, the yield behavior during machining slightly depends on the rate of deformation. As the strain and/or strain rate increases, the cutting resistance also increases since the shear zone gets smaller and deformation becomes restricted to a smaller region [3].

$\phi=45+\frac{\alpha}{2}-\frac{\beta}{2}$

Crystallographic differences have been found during deforming crystalline solids based on experimental observations on tensile deformation of single crystals $[4,7]$. The deformation in single crystals is along closed-pack directions in closed-pack planes. FCC metals have 12 possible slip systems composed of three directions on four distinct planes. Figure 1 shows a crystal with 12 closed-pack directions, cutting direction (CD) aligned with the [010] direction, and the cutting plane (CP) being in (100) plane. Simple shear deformation on the shear plane (SP) is shown with the movement of material from position $\mathrm{ABCD}$ to $(\mathrm{ABCD})$ '. The examples in the literature for planing operation use the transverse direction (TD) being as fixed at certain angles while the crystal being machined.

There have been numerous studies done on micromachining investigating crystallographic effects on single crystals. Black and Cohen $[8,9]$ carried out ultra-micro-

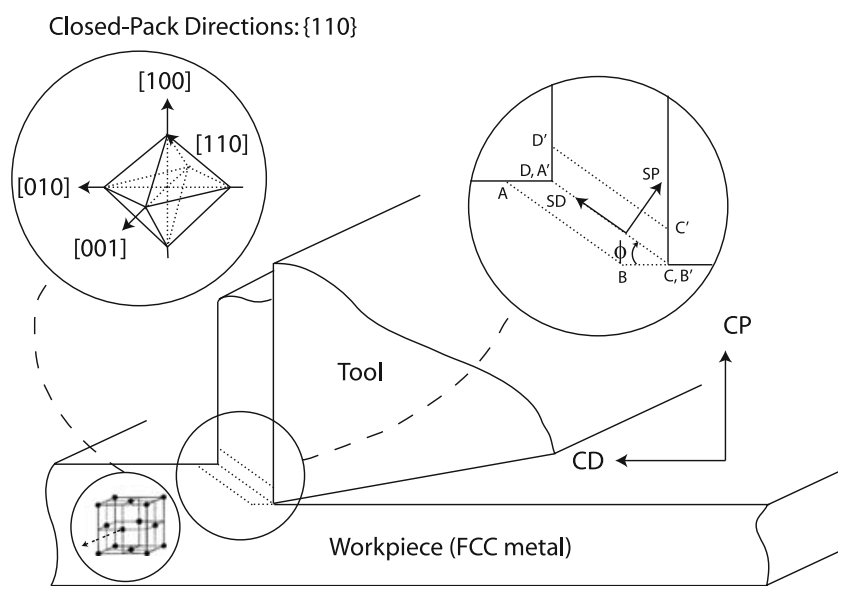

Fig. 1 Machining a FCC material with shown crystal orientation tomy tests on single-crystal copper and aluminum for measuring forces and shear angles. An important experimental observation is the shear angle and cutting forces changing with a four-fold symmetry for TD being in the [001] direction. Ueda performed experiments on two-phase single-crystal material, $\beta$-brass, and investigated the chip morphology for each orientation [13] and observed two different types of chips; fractured and lamellar. The specific energy of for machining has changed as the workpiece orientation varied. The forces observed fluctuate more with fracture in chips and forces become smoother as the chips are formed in more regular, lamellar form. Facing experiments on single-crystal CaF by Yan have illustrated [14] the periodical variations in the quality of machined surface with changing crystal orientation over the cross section of the material. Koenig and Spenrath obtained poor surface quality when machining single-crystal copper in the [100] direction but better surface quality for the [110] direction and with less force variation [10]. Williams and Horne found the shear stress to be $40 \%$ lower in the machining workpiece having the (111) plane as the cutting plane than having the [111] direction as the cutting direction [11]. According to these studies, the quality of the product is strongly dependent upon the crystallographic orientation of the material.

Sato [12] has carried out two sets of experiments on aluminum: one of which has $\mathrm{CD}$ and $\mathrm{CP}$ both lying in the (001) plane with varying set of angles $(\beta)$ about the [001] direction, and the other orientation is for the (110) plane. The Schmid-based model is applied to describe the fluctuation of forces. The model uses the measured resultant forces as inputs and resolves to 12 slip systems. The thrust and cutting forces are resolved into slip systems. The amount of slip on each slip system is assumed to be proportional to the amount of resolved shear stress on that slip system, contrary to the Schmid theory. ${ }^{1}$ SEM images have shown that three types of deformation zones exist in the shear plane and in each zone different slip systems are active. This suggests the necessity of a model taking into account crystal rotation during deformation. During the machining of cold-rolled aluminum in various angles to its rolling direction, differences in shear angles are observed.

The Taylor plasticity model has been applied to the machining of single crystals by Lee, Zhou, and Ngoi, considering multiple slip for single crystals $[15,23]$. Forces

\footnotetext{
${ }^{1}$ The active slip systems are the ones that exceed the critical value of shear stress when the applied stress is resolved to the 12 slip systems according to Schmid.
} 
are calculated through estimated shear angle and assuming shear plane solution. The Taylor factor variation for turning a single-crystal workpiece about a crystallographic direction is estimated and compared to experimental results. Due to the ambiguity in finding a minimum work solution to find a shear angle, the texture softening factor (TSF) concept is introduced to minimize the work done on the crystal. The model of Arcona and Dow is similar to Lee's work, which is also based on the perfectly plastic material model but includes the effects of material elasticity [26].

The edge radius of the tool has an increasing effect on surface quality, subsurface damage, and the specific energy of a cut as the depth of cut gets smaller [27]. Lucca developed a sliding indentation model and estimated the subsurface damage for aluminum to be $0.31-0.81 \mu \mathrm{m}$ for depth of cuts of $0.1-1 \mu \mathrm{m}$, respectively, which are very close to the X-ray results [28]. Moriwaki and Okuda [25] observed that below $0.2 \mu \mathrm{m}$ depth of cut, rubbing and burnishing become more dominant than shear and no crystallographic effects are observed during machining polycrystal copper.

MD simulations provide some understanding for chipformation mechanics at small scales and crystallographic effects. The chip formation, surface quality, subsurface damage, and side spread are observed to be very much dependent upon crystal orientation [32, 33]. When the [110] direction is in the shear plane, the deformation is observed to be more restricted to shear plane and the surface quality is better when compared to the other orientations.

In this paper, a Taylor-based rate-insensitive crystal plasticity model is proposed for better understanding the crystallographic effects when machining single-crystal materials also considering friction tool and the workpiece. Edge radius and initial dislocation content of the raw material, depending on its strain history, limit the observation of crystallographic effects during machining. If these effects are minimized by the use of a sharp diamond tool and well-annealed workpiece, then a constitutive crystal plasticity model would be effective in estimating the specific energy and shear angle for a given workpiece orientation for orthogonal machining. In the proposed model, one can assign different critical resolved shear $\operatorname{stresses}\left(\tau_{c r}\right)$ to different slip systems. For the simplicity of analysis, a value of $\tau_{c r}$ is assumed for each slip system. Hence, the proposed model is more suitable for materials having a low latent hardening coefficient like for aluminum, where $\tau_{c r}$ does not change significantly from one slip system to another unless there is texture in the material.

The machining model could be applied to two main orthogonal cutting operations, planing and turning. In addition, the Taylor factor variation for orthogonal machining is shown for various crystal orientations in Euler space. The frictionless cases are compared to the result of a case with a coefficient of friction of 0.3 between the tool and workpiece.

\section{Modeling}

In this paper, Taylor-based crystal plasticity code in MatLab is written for the machining operations. The results of Taylor's model were checked with the Bishop-Hill(BH) work maximization principle. The Schmid model and its applicability to machining process is also discussed in the following section.

\subsection{Schmid-based models}

In previous studies, Schmid-based models were applied to find the shear angle by using measured thrust and cutting forces and resolving them into slip systems. There are two major drawbacks of these models. One is during finding the shear angle; Sato assumed the amount of slip proportional to the amount of $\tau_{R S S}$, which is not in agreement with Schmid theory. ${ }^{2}$ Second, assuming the material simply under a principal stress state is an even rougher assumption than the shear plane assumption, where machining is described with a shear stress and a normal stress at the shear plane (Eq. 3). This is simply because when the stress state at the shear plane is transformed to sample frame, the resulting state would not necessarily be a principal stress state having no shear stress components. A sample stress state at the shear plane:

$$
\sigma^{\text {shear }}=\left(\begin{array}{ccc}
0 & 0 & \tau_{s} \\
0 & 0 & 0 \\
\tau_{s} & 0 & \sigma_{n}
\end{array}\right)
$$

A method that can be used to find the shear angle using the Schmid model is described as follows. The model needs shear and normal stresses at the shear plane that can be experimentally determined from forces and the shear angle. In addition, the model needs a priori knowledge of $\tau_{c r}$, since the known stress state at the shear plane for a given crystal orientation makes the Schmid model applicable. The shear angle for a crystal orientation then can be found in seven steps: i. Assume a shear angle between 1 and $88^{\circ}$; ii. Transform the stress state from shear

\footnotetext{
${ }^{2}$ The active slip systems are the ones that exceed the critical value of shear stress when the applied stress is resolved to the 12 slip systems according to Schmid.
} 
plane to sample and then to crystal frame; iii. Resolve the stresses into 12 slip systems using Eq. (4); iv. Assume a $\tau_{c r}$ and identify the active slip systems exceeding that critical value of stress; v. Sum the all the active slip vectors to get the resultant slip vector; vi. Transform the resultant vector from crystal frame to sample frame; vii. The shear angle can then be found by first taking the inner product of the resultant vector with the cutting direction and cutting plane vector separately and then by the taking inverse tangent of resolved components in cutting direction and plane. A general method is preferred since the resultant vector could be a spatial vector and simply projecting would yield a spatial angle rather than shear angle defined in shear plane assumption.

$\tau_{R S S}=b_{i} \sigma_{i j} n_{j}$

\subsection{The Taylor-based model}

This model is based on Taylor's multiple slip analysis for single crystals. The fundamental relationship between shearing of a material and the crystal orientation of the material can be elucidated through a Taylor-based method without introducing the complications from a rate-sensitive model. Besides, the results of rate sensitive models would not be much different since the minimum work could be achieved by activating minimum number of slip systems.

Taylor models determines the amount of strain along each of the five independently selected slip systems by minimizing the sum of internal work done in the crystal for the desired deformation. There are two inputs to the model: i. the deformation gradient, which has the effect of amount of strain, and the effect of rake angle; ii. Crystal orientation with respect to the direction of cutting.

The rate-insensitive methods posses an ambiguity problem in selecting the active slip systems. However, a unique solution can be found for the determination of Taylor factors or the amount of work, which is of our interest. Slip is a simple shear type of deformation in closed-pack planes at closed-pack directions in FCC metals [6, 7]. For the case of machining, the deformation gradient is the same as slip, simple shear. Hence, work minimization always boils down to the problem of finding the closed pack directions along in the nearest to machining shear direction. In the case of a non-symmetric deformation gradient with respect to crystal orientation, deformation is higher as more slip systems are activated. This shows the importance of this study over rate-sensitive methods since the relation between crystal orientation and work required is correlated with simplified relationships.
The shear angle and the shear stress at the shear plane is calculated by minimizing the crystallographic work in a range of possible shear angles for a given crystal orientation. The shear angle would be the one that yields the minimum amount of work among the possible range of $1-88^{\circ}$.

Shear-plane solution is assumed in the model in which the possible shearing of the workpiece occurs in a single plane. In plane strain orthogonal cutting, the deformation gradient (DG) in the shear plane (SP, shown in Fig. 1), consists of two parts: the shear-strain part $(\epsilon)$ and the rotation part $(\Omega)$ with a superscript "shear" indicates the state belonging to the shear frame.

$D G^{\text {shear }}=\epsilon^{\text {shear }}+\Omega^{\text {shear }}$

$D G^{\text {shear }}=\left(\begin{array}{ccc}0 & 0 & \gamma \\ 0 & 0 & 0 \\ 0 & 0 & 0\end{array}\right)=\left(\begin{array}{ccc}0 & 0 & \frac{\gamma}{2} \\ 0 & 0 & 0 \\ \frac{\gamma}{2} & 0 & 0\end{array}\right)+\left(\begin{array}{ccc}0 & 0 & \frac{\gamma}{2} \\ 0 & 0 & 0 \\ -\frac{\gamma}{2} & 0 & 0\end{array}\right)$

Taylor's model uses the symmetric part of the DG that is the strain part as an input only. The shear strain then has to be transformed from shear plane (SP) into workpiece coordinates or sample frame through an assumed shear angle of $\phi$ through the transformation $g^{\text {sh2samp }}$.

$$
\begin{aligned}
& g^{\text {sh2sam }}=\left(\begin{array}{ccc}
\cos \phi & 0 & -\sin \phi \\
0 & 1 & 0 \\
\sin \phi & 0 & \cos \phi
\end{array}\right) \\
& \epsilon_{i j}^{\text {sample }}=g_{i k}^{\text {sh2samp }} g_{j l}^{\text {sh } 2 \text { samp }} \in_{k l}^{\text {shear }}
\end{aligned}
$$

Strain has to be transformed into crystal frame from sample frame via the transformation rule as shown above. For a given unit, cutting direction of [uvw] and cutting plane of (hkl), the passive transformation matrix, can be written as;

$g^{\text {samp } 2 c r y}=\left(\begin{array}{ccc}u & o & h \\ v & p & k \\ w & q & l\end{array}\right)$

Similarly, the strain in the crystal, $\varepsilon^{c}$, becomes;

$\in_{i j}^{c}=g_{i k}^{\text {samp } 2 c r y} g_{j l}^{\text {samp } 2 c r y} \in_{k l}^{\text {sample }}$

The slip directions and corresponding slip planes for FCC metals are shown in the following table. 
Slip Plane, $\mathbf{n} \quad$ (111)

(111)

(111)

(11) 1$)$

Slip Direction, b

$[01 \overline{1}]$

$[\overline{101}] \quad[1 \overline{1} 0]$

$[0 \overline{11}]$

[101]

$[\overline{1} 10]$

$[01 \overline{1}$

$[101]$

$[\overline{110}]$

$[0 \overline{11}$

$[101]$

$[110]$

The work done on the crystal can be estimated by two means. One way is to compute work by the microscopic shear rates, $\Gamma$, on slip systems having slip resistances of $\tau_{c}$ [4]. The other is the estimation of work in macroscopic perspective by multiplying the strain that the crystal is exposed to with the stress states that the crystal can posses during slip [5].

$\delta w=\sigma \in^{c}=\tau_{c} \Gamma$

The five slip systems must be independent from one another to result in a solution. According to Taylor, multiple slip deformation is carried out with five active slip systems among 12 possible ones for FCC metals. The sum of the diagonal components of strain vanish according to volume constancy assumption during deformation. Hence, one dependency on six unknown components of symmetric strain tensor are removed, yielding five unknowns to be determined overall. A five-by-five coefficient matrix is formed to find five unknowns of strain for five slip systems. If there is dependency among the selected five slip systems, then a unique solution cannot be determined. The determinant of the coefficient matrix will be zero if there is any dependency among slip systems among 792 combinations.

$\epsilon^{c}=\sum_{\alpha=1}^{5} m^{\alpha} \delta \gamma^{\alpha}$

$m^{\alpha}=b^{\alpha} \otimes n^{\alpha}$

The active slip systems during deformation will be the ones that yield the minimum through Eq. (14).

$\delta w=\tau_{c} \sum_{i=1}^{5} \delta \gamma$

In general, if there is symmetry between the orientation of the crystal and the deformation gradient, there is more than one minimum solution. The reason for this is the shear becoming zero for some of the slip of the five systems among minimum work solutions. The slip system with zero slip can be any alternative among the 12 that is independent from the other previously selected slip systems. Hence, ambiguity arises for symmetric orientations for machining.
Taylor factor (M) is a measure of the crystal for a given orientation and deformation. It is the ratio of the applied stress to critical resolved shear stress. The $\tau_{c}$ term in Eq. (13) could be taken outside the summation, assuming both equal the amount of shear stress requirement to activate the slip systems and equal hardening for each of the 12 slip systems.

$M=\frac{\sigma}{\tau_{c}}=\frac{\sum_{i=1}^{5} \delta \gamma}{\epsilon_{v m}}$

For machining, the work done by the slip deformation is equivalent to the work done on the shear plane $\left(\epsilon_{v m}=\frac{\gamma}{2}\right.$ and $\sigma=\tau_{\text {shear }}$ ). The Taylor factor then becomes;

$M=\frac{\sum_{i=1}^{5} \delta \gamma}{\frac{\gamma}{2}}=\frac{\delta w}{\tau_{c} \frac{\gamma}{2}}$

The macroscopic work done is only at the shear plane according to shear-plane assumption;

$\delta w=\tau_{\text {shear }} \frac{\gamma}{2}$

The shear stress on the shear plane, $\tau_{\text {shear }}$, can be related to the Taylor factor through macroscopic to microscopic work equality.

$M=\frac{\tau_{\text {shear }}}{\tau_{c}}$

Making an assumption for the normal stress at the shear plane being as one-third of the hardness $(\mathrm{H})$, the specific energy of cut $\left(K_{c u t}^{s p}\right)$ can be estimated [26]. The cutting force (Fc) can be calculated with knowing both shear angle and shear and normal stresses at the shear plane. Specific energy of cutting is the amount of energy required to cut a unit of material (d: depth of cut, w: width of cut). It should be noted that friction and rake angles could also be included in the cutting force definition.

$F c=K_{c}^{s p}(d w)=\left(\tau_{\text {shear }} \cos \phi-\frac{H}{3} \sin \phi\right) A_{\text {shear }}$

where shear area is

$A_{\text {shear }}=\frac{d w}{\sin \phi}$

Rearranging the terms, the specific energy becomes

$K_{c}^{s p}=\frac{\tau_{\text {shear }}}{\tan \phi}-\frac{H}{3}$ 
or

$K_{c}^{s p}=\frac{M \tau_{c}}{\tan \phi}-\frac{H}{3}$

To estimate the specific energy by minimizing the sum in Eq. (15), the shear angle first has to be found over a range of possible angles. The shear stress on the shear plane changes proportionally with the Taylor factor (Eq. 18) since critical resolved shear stress is assumed to be a constant. Knowing the shear angle and corresponding minimum Taylor factor, specific energy of cutting can be estimated.

Up to this point, the model is parallel with Lee and Zhou's work [16]. Apart from the presented work, they used texture softening factor (TSF) as a means of finding a unique solution among possible solutions. TSF is based on the fact that the crystal would rotate in the direction that the multiplication of net rotation and the Taylor factor change with respect to initial orientation is smallest. In general, it appears to be in the directions where the Taylor factor change is negative, called "softening". This second-order minimization makes sense and is useful in determining textures formed in rolling where the crystal is free to rotate in any direction. However, it is not suitable for machining, because rotation, the skew-symmetric part of the deformation gradient, is already defined in the machining process as given in Eq. (3). In other words, the crystal is not free to rotate in any direction where TSF is minimum, rather, the crystal is forced to be rotated in a specific direction. Besides, in their work, according to their notation, TSF is supposed to be a second-order tensor rather than a scalar, since the derivative with respect to the second-order tensor yields another second-order tensor by definition.

In addition to TSF, they have used effective Taylor factor $\left(M_{e f f}\right)$ as a means of compensating the drop in shear strain as the shear angle diverges from $45^{\circ}$, an amount of $\psi$ similar to Merchant's principle. The value of the shear strain given to the crystal increases with $\cos 2 \psi$. Since shear strain is independent of the shear angle (see Fig. 2), there is no need to compensate for the shear strain. On the other hand, the possible low-shear-angle solutions experimentally observed during machining single crystals are eliminated.

$M_{e f f}=\frac{M}{\cos 2 \psi}$

In this paper, the change of Taylor factor $(\mathrm{dM})$ is used to explain chip formation behavior during machining and is calculated through the rotation part of the imposed deformation gradient on the shear plane. The Taylor factor before the rotation of the crystal and after the rotation is estimated and the difference is used to calculate the change (dM). Actually, it is based on the assumption that all the rotation of the lamella is elastic, forming a texture rather than being plastic, since the crystal orientation does not change with plastic deformation. This change could be used to explain the hardening or softening behavior during single lamella formation.

$d M=M_{\text {after }}-M_{\text {before }}$

Crystal orientation has a significant effect on the specific energy of a cut for certain orientations in which deformation is not aligned with one of the closed-pack directions. To illustrate this fact, shear strength is shown for various orientations in Euler space including the symmetric ones. In order to set an orientation, Bunge angles of $0 \leq \phi_{1} \leq 2 \pi, 0 \leq$ $\theta \leq \pi, 0 \leq \phi_{2} \leq 2 \pi$ are used that are rotations about Z, X', Z', respectively.

\section{Results and discussion}

Turning and orthogonal planing of single-crystal samples is studied such that the angle around TD is varied and TD is a common crystallographic direction like in many of the experiments done in literature [18-22, 24, 29, 31].

Orthogonal planing in an orientation such that TD being in the [001] direction is shown in Fig. 3 with closed-pack directions and closed-pack planes. The crystallographic slip is a simple shear type of deformation just as is the shearing deformation at shear plane for machining.

The Taylor factor is found to be a constant when machining a crystal as shown in Fig. 4. The reason for this is that there is always a closed-pack direction (CPD) aligned with the deformation gradient for this cutting orientation. As mentioned previously, the shear angle is chosen as the one that yields minimum amount of work. For each orientation, it is the same since the minimum occurs along the closed-pack direction and there is always one closed-pack direction in between the shear angles of $1^{\circ}$ and $88^{\circ}$. By looking at the shear angle plots in Fig. 5, the change of shear angles with the change in orientation can easily be correlated. The shear angle is the same angle between $C D$ and the 110 closed-pack vector in the crystal, so, as the crystal rotates, the shear angle changes and makes a big jump until the angle between CPD and CD exceeds $90^{\circ}$, after which another CPD shows up and takes care of

Fig. 2 The strain during shearing does not vary with shear angle

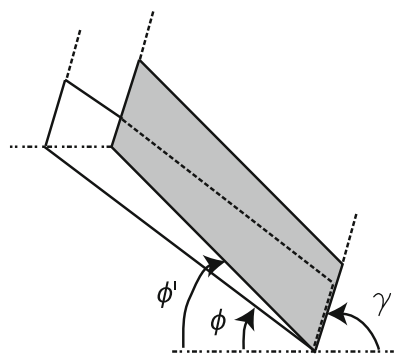



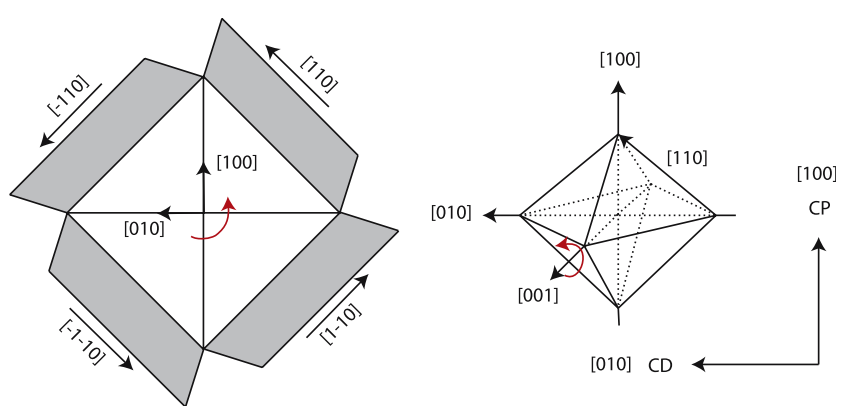

Fig. 3 Closed-pack directions for TD in the [100] direction

the deformation. This way, four periodicity is observed. The resulting shear angle is as high as $90^{\circ}$ since there is no friction angle contribution.

The difference in forces when planing about the [001] direction can be explained with the changes in specific energy. Due to large jumps in shear angle from $0^{\circ}$ to $90^{\circ}$, the specific energy value becomes quite large (Eq. 23) and makes cutting forces higher when cutting $\mathrm{CD}$ is in the [110] direction. The specific energy is lowest when the $\mathrm{CD}$ is selected as [100] directions.

The $\mathrm{dM}$ plots give information about the hardening or softening behavior during chip formation. In addition, it could be used to explain the very low shear angles observed during machining single crystals. The negative values indicate ease in chip formation. During machining, the shear angle becomes lower with the aid of friction. In this case, the actual shear angle could even then become the one with higher Taylor factor, which demands higher work but with a softening rate. This can be used to explain the ease

a
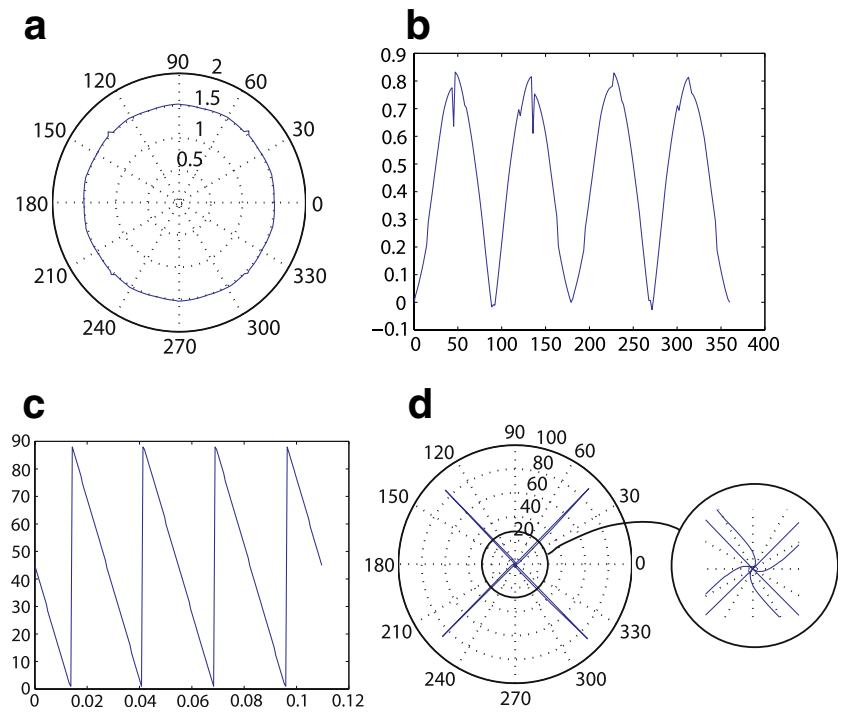

Fig. 4 a Taylor Factor. b Taylor Factor change as crystal rotates (dM) c Shear angle. d Specific energy without friction for TD being in the [100] direction

in chip formation when $\mathrm{CD}$ is in the [100] direction, whereas fractured discontinuous chip are formed when $\mathrm{CD}$ is in the [110] direction [30].

Friction lowers the shear angle and raises the Taylor factor (Fig. 5). Higher Taylor factors belong to the orientations where the deformation is carried out activating more number of slip systems. A four-fold symmetry is observed in cutting with friction in agreement with experimental observations for this orientation $[8,9]$.

As the orientation becomes more symmetric, the Taylor factor plot becomes smoother because of the higher availability in the number of slip systems. The same analysis is performed for TD being in the [110] direction and the results are shown in Fig. 6. Two sets of equally stressed slip systems become active during the deformation. Around the [110] direction, the Taylor factor remains constant but the shear angle varies due to switching from one couple of slip systems to another. The specific energy does not seem to be symmetric but the difference in peaks is due to the division of small numerical differences becoming amplified by near-zero shear angles (Eq. 21). At smaller values of specific energies, the resultant pattern is symmetric. The dM plot shows no symmetry and it is noisy because of the uniqueness problem introduced with the Taylor method that yields many alternative solutions. It could be the same reason of the noisy (or large fluctuations in) shear angle and force results in the model of Lee and coworkers.

Similarly, the Taylor factor variation and corresponding "dM" change is calculated for cutting around the (110) plane. Taylor factor again remains constant for every angle around the [110] direction, but the "dM" changes significantly, changing the nature of deformation. "dM" takes negative or positive values depending on the orientation.

Using the same method, orientation effects without friction for turning around the [100] axis is modeled. Note in turning that the cutting plane always remains constant, whereas the cutting direction changes direction during full revolution of the workpiece in contrast to planing [17]. The resulting Taylor factor variation over one revolution of the

a
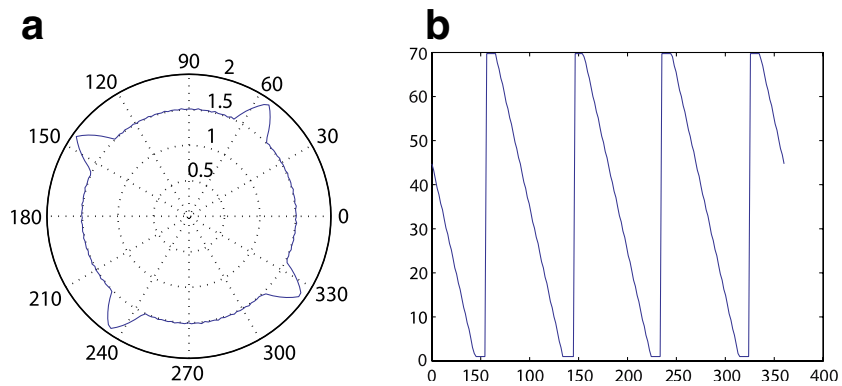

Fig. 5 a Taylor factor and $\mathbf{b}$ shear angle results with assumed friction coefficient of 0.33 for TD being in the [100] direction 
a

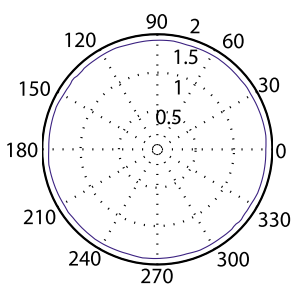

C

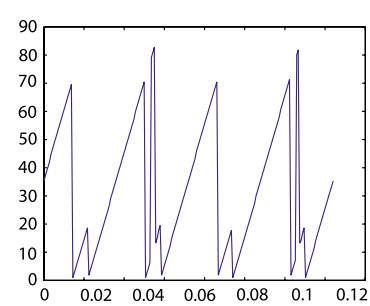

$\underset{0.9}{\mathbf{b}}$

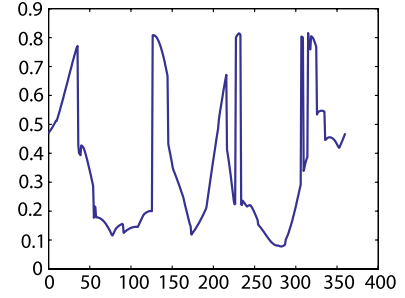

d

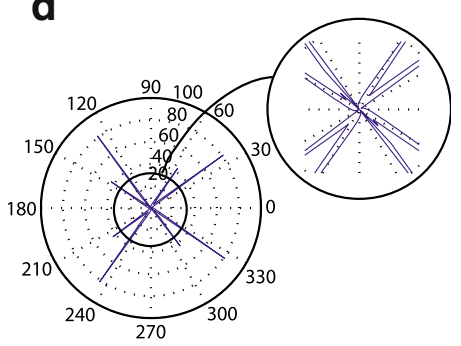

Fig. 6 a Taylor factor. b Taylor factor change as crystal rotates (dM). c Shear angle. d Specific energy without friction for TD being in the [110] direction

workpiece that is due to the change of crystal orientation is shown in Fig. 7. Fluctuation of Taylor factor is rather remarkable since the orientation is no longer symmetric with respect to the input deformation gradient.

The Taylor factors are estimated for every orientation using Euler angles for without friction case (Fig. 8). This orientation plots could be used for any orthogonal machining case, not only for planing but also for turning for a

a

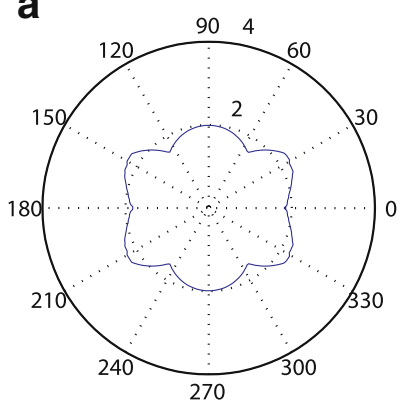

C

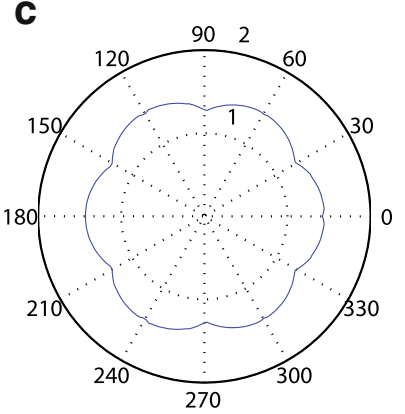

b

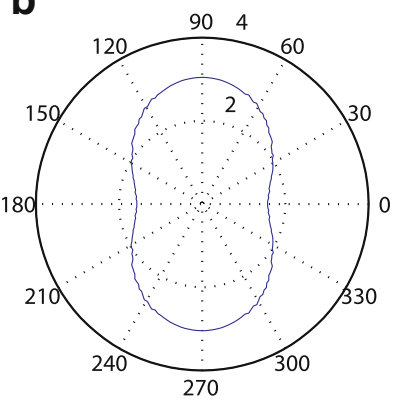

d

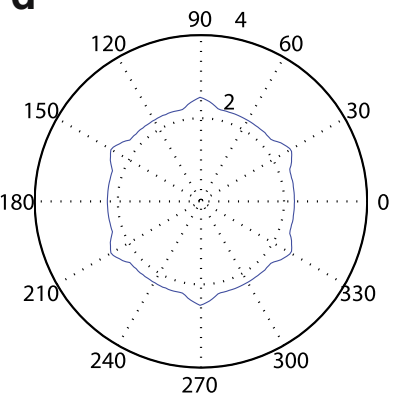

Fig. 7 Results for turning. a Taylor factor for a CP of (100). b Effective Taylor factor (provided for comparison with literature results [17]) for a CP of (100). c Taylor factor for a CP of (110). d Effective Taylor factor (provided for comparison [17]) for a CP of (110)

given $\mathrm{CD}$ and $\mathrm{CP}$, by the method shown in the previous section to find orientation angles. Each point on the surface belongs to a co-latitude $\left(\phi_{1}\right)$ and longitude $(\Phi)$ specified by two Euler angles. The other angle $\left(\phi_{2}\right)$ represents the amount of rotation around the vector defined by the former two angles, which is kept constant against $\phi_{1}$ and $\Phi$.

The Taylor factor for the frictionless case has its maxima at the symmetric orientations. However, in the case minimums, the deformation gradient is symmetric with respect to the given orientation and the Taylor factor becomes minimum since the minimum number of slip systems are activated. In-between multiple slip orientations exist in which the deformation is not symmetric with any of the closed-pack directions. An interesting note could be for $\phi_{2}$ being around $90^{\circ}$, the Taylor factor variation stabilizes and changes are very small compared to other orientations $\left(\phi_{2}=99.3^{\circ}\right.$ and $\left.\phi_{2}=74.5^{\circ}\right)$. These orientations might be
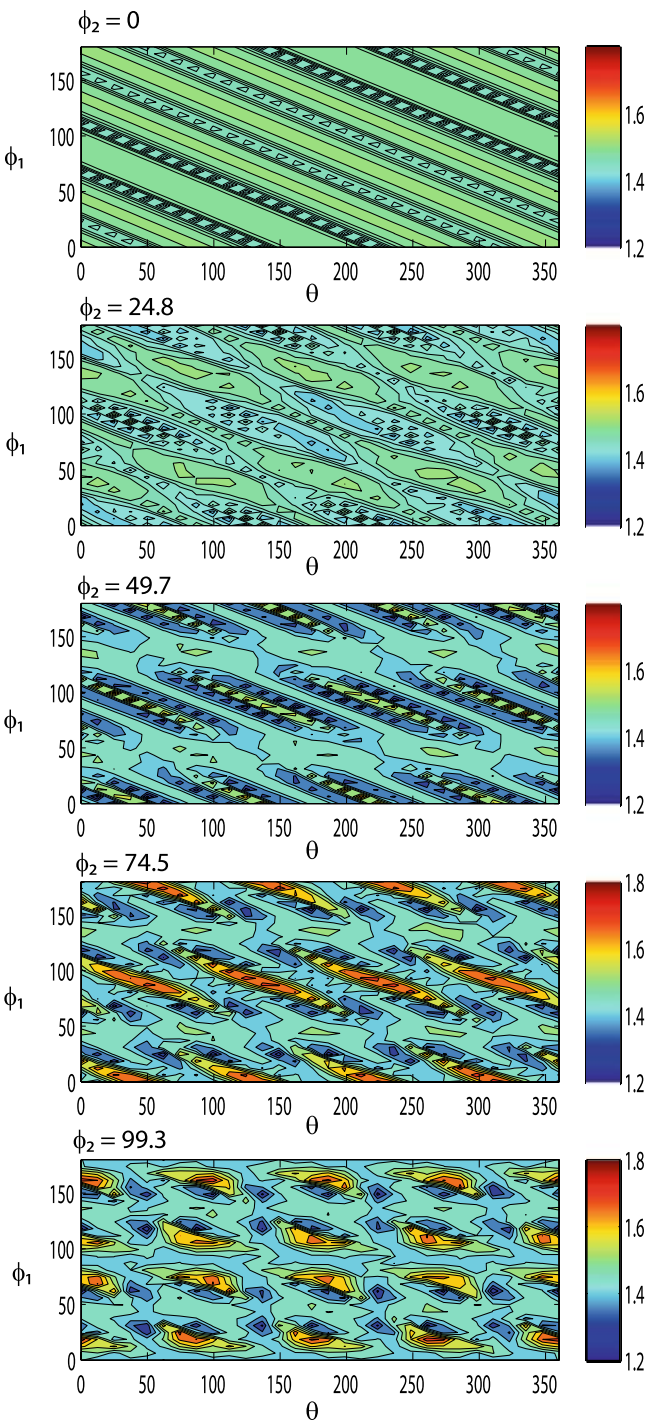

Fig. 8 Taylor Factor for orthogonal machining for every orientation $\left(\phi_{1}, \theta, \phi_{2}\right)$ without friction 
chosen to have a stable cut to have smooth surfaces. Each orientation plot posses symmetry and strong dependence on the crystallography of the material being processed.

The case with the friction is shown in Fig. 9 for the same half of the orientations of the case with the friction since the other half is symmetric. Taylor factor values increase on average when the case with friction is compared to the case without friction. This is due to the lower number of closedpack directions becoming available during cutting with the introduction of friction. The friction angle forces multipleslip deformation for some orientations, so it increases the crystallographic work done. In the case of friction for certain orientations, peaks appear, and the smooth distribution in the frictionless case no longer exists.

$\phi_{1}$
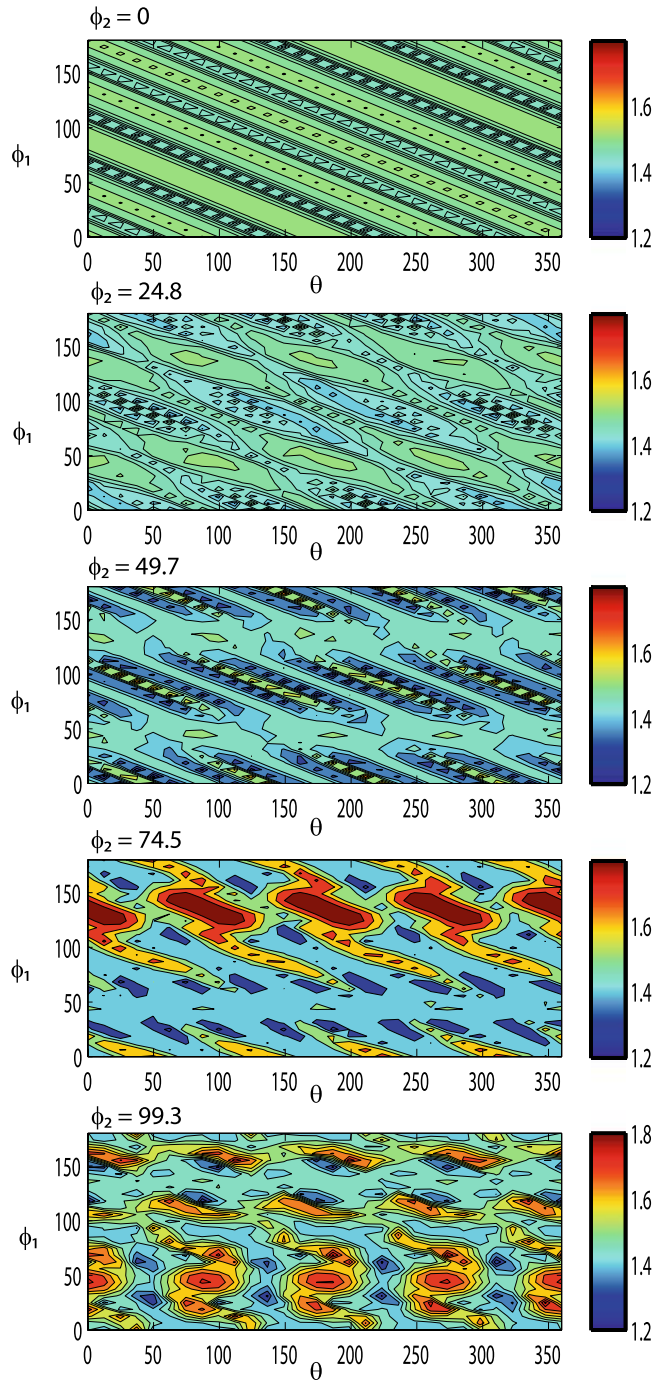

Fig. 9 Taylor factor for each orientation $\left(\phi_{1}, \theta, \phi_{2}\right)$ with a friction coefficient of 0.33

\section{Summary and conclusions}

The crystallographic effects on machining performance are investigated from a modeling point of view in this research. Apart from the literature, specific energy of cut is related to the Taylor factor and the usefulness of effective Taylor factor and texture softening factor is discussed. Orthogonal cutting, a plain strain case, is modeled by using the Taylor plasticity model. The solution becomes the closed-pack direction nearest the deformation gradient, since slip and machining deformation are of both simple shear type. Some important conclusions can be listed as follows:

- The Taylor factors and shear angles for TD being [100] is calculated. The deformation gradient is in line with the two closed-packed directions where slip deformation is also of the simple shear type. Therefore, the work done on the crystal is invariant of the rotation about [100]) as well as [110]. A four-fold symmetry is observed in the shear angles found for the TD being in [100] for the planing case like in the experiments of J. T. Black.

- Again four-fold periodicity during planing can be observed with the introduction of friction angle to Taylor's crystal plasticity, causing more slip systems to become active. The differences get smaller as the plane of orthogonal cutting happening becomes more symmetric $(100) \rightarrow(110) \rightarrow(111)$.

- For turning operations, many slip systems are activated, leading to a higher amount of Taylor factor and so specific energies.

- According to the Taylor-based model, the shear angle can have values as high as $90^{\circ}$, which is not reasonable. Hence, the effect of friction has to be included in the model. This is achieved by selecting the result with next smallest work, but yielding a lower shear angle. It should be noted that the shear stress in this case varies.

- The usefulness of effective Taylor for machining is questionable. It is used as a means of lowering the shear angle from $90^{\circ}$ although, it does not allow very low shear angles that are experimentally observed.

- The use of texture softening factor(TSF) in finding a unique solution is also questionable. TSF, through minimization of a second-order of work, used to tell which direction the crystal would rotate for a given orientation for rolling operation. In machining, however, the rotation part of the deformation gradient, $\Omega$, is defined.

Acknowledgements I had the pleasure of taking the class "Advanced Characterization of Materials" given by a great person and outstanding teacher A.D. Rollett in CMU. The invaluable discussions with Prof. 
Ozdoganlar and his orientations and ideas are also greatly appreciated. I am also thankful to my father and mother, Necdet and Tulay Demir, for their endless encouragement motivating this project.

\section{References}

1. Shaw MC, Finnie I (1955) Shear stress in metal cutting. Trans ASME, pp 115-125

2. Van Luttervelt CA, Childs THC, Jawahir IS, Klocke F, Venuvinod PK (1998) Present situation and future trends in modeling of machining operations. CIRP 47:587-636

3. Manjunathaiah J, Endres WJ (2000) A new model and analysis of orthogonal machining with an edge-radiused tool. Trans ASME 122:384-390

4. Taylor GI (1938) Plastic strain in metals. Inst Metals 62:307-325

5. Bishop JFW, Hill R (1951) A theory of plastic distortion of a polycrystalline aggregate under combined stresses. Phila Mag 42:1298-1307

6. Reid CN (1973) Deformation geometry of materials, 1st edn. Pergamon Press, Oxford

7. Kocks UF, Tome CN, Wenk HR (1998) Texture and anisotropy. Cambridge University Press, Cambridge

8. Cohen P, Black JT (1984) Strain, strain rate and shear velocity measurements in metal cutting. High Energy Rate Fabrication. ASME pp 271-278

9. Black JT (1979) Flow stress model in metal cutting. J Eng Ind 101:403-415

10. Koenig W, Spenrath N (1991) Influence of crystallographic structure of the substrate material on surface quality and cutting forces in micromachining. Proceedings of the International Precision Engineering Seminar, pp 141-151

11. Williams JA, Horne JG (1982) Crystallographic effects in metal cutting. J Mater Sci 17:2618-2624

12. Sato M, Kato Y, Tsutiya K, Aoki S (1980) Effects of crystal orientation on the cutting mechanism of aluminum single crystal. Bull JSME 24(215):1864-1870

13. Ueda K, Iwata K, Nakajama K (1980) Chip formation mechanism in single-crystal cutting $\beta$-Brass. Ann CIRP - Manufacturing Technology 29(1):41-46

14. Yan J, Syoji K, Tamaki J (2004) Crystallographic effects in micro/ nanomachining of single-crystal calcium fluoride. J Vac Sci Technol B22(1):46-51

15. Lee WB (1990) Prediction of microcutting force variation in ultraprecision machining. Precis Eng 12(1):25-28
16. Zhou M, Ngoi BK (2001) Effect of tool and workpiece anisotropy on microcutting processes. IMechE J Eng Manuf 215:13-19

17. Lee WB, Cheung CF, To S (2002) A microplasticity analysis of micro-cutting force variation in ultra-precision diamond turning. Trans ASME 124:170-177

18. Lee WB, Chan KC (1990) Symmetry requirement in shear band formation. Scr Mater 24:997-1002

19. Lee WB, Zhou M (1993) A theoretical analysis of the effect of crystallographic orientation on chip formation in micromachining. Int J Mach Tools Manuf 33(3):439-447

20. Lee WB, To S, Sze YK, Cheung CF (2003) Effect of material anisotropy on shear angle prediction in metal cutting - a mesoplasticity approach. Int J Mech Sci 45:1739-1749

21. Lee WB, To S, Cheung CF (2000) Effect of crystallographic orientation in diamond turning of copper single crystals. Scr Mater 42:937-945

22. Lee WB, Cheung CF (2001) A dynamic surface topography model for the prediction of nano-surface generation in ultraprecision machining. Mech Sci 43:961-991

23. Lee WB, To S, Cheung CF (2003) Friction-induced fluctuation of cutting forces in the diamond turning of aluminum single crystals. IMechE J Eng Manuf 217:615-631

24. Ohmori G, Takada S (1982) Primary factors effecting accuracy in ultra-precision machining by diamond tools. Bull Jpn Soc Precis Eng 16(1):3-7

25. Moriwaki T, Okuda K (1989) Machinability of copper in ultraprecision micro diamond cutting. Ann CIRP 38(1):115-118

26. Arcona C, Dow TA (1998) An empirical tool force model for precision machining. Trans ASME 120:700-707

27. Rubenstein C, Lau WS, Venuvinod PK (1985) Flow of workpiece materials in the vicinity of the cutting edge. Int J Mach Tool Des Res 25:91-97

28. Lucca DA, Seo YW (1994) A sliding indentation model of the tool-workpiece interface in ultra-precision machining. Tribology Symposium ASME, pp 17-22

29. Liang Y, Moronuki N, Furukawa Y (1994) Calculations of the effect of material anisotropy on microcutting process. Precis Eng 16:132-138

30. Iwata K, Osakada K, Terasaka Y (1984) Process modeling of orthogonal cutting by the rigid-plastic finite element method. Trans ASME 106:132-138

31. Wince JN (2002) Modeling chip formation in orthogonal metal cutting using finite element methods. Master's Thesis, Mississippi State University, Mech Eng. Dept

32. Ikawa N, Shimada S, Tanaka H (1992) Minimum thickness of cut in micromachining. Nanotechnology 3:6-9

33. Komanduri R, Chandrasekaran N, Raff LM (1999) Orientation effects in nanometric cutting of single-crystal materials an MD simulation approach. Ann CIRP, pp 67-72 